\title{
Charge Ordering and Phase Separations in the Molecular Crystal Model
}

\author{
W.R. Czart*, P.R. Grzybowski and S. Robaszkiewicz \\ Faculty of Physics, A. Mickiewicz University, Umultowska 85, 61-614 Poznań, Poland
}

\begin{abstract}
We analyse the ground state phase diagrams and thermodynamic properties of charge orderings in narrow band materials using the molecular crystal model in the static limit. We present results for the hypercubic lattices in dimensions $d=2$ and $d=\infty$. We focus our study on the problem of phase separations involving charge orderings and the effects of next-nearest-neighbor hopping $\left(t_{2}\right)$ on the charge ordered states in these systems. The ground state phase diagrams are evaluated for a few representative cases. Results for the molecular crystal model are compared with those obtained previously for the spinless fermion model with repulsive intersite interaction $W$.
\end{abstract}

PACS numbers: 71.10.Fd, 71.30.+h, 71.45.Lr, 64.75.Gh

\section{Introduction}

There is ongoing, intense, research (see e.g. [1-14] and references therein) in the field of electron charge orderings $(\mathrm{CO})$ and charge density waves phenomena, due to experimental discovery of such phases in manganites, cuprates, numerous organic conductors and many other important materials. Two main mechanisms are invoked in explaining $\mathrm{CO}$ phenomena: electron correlations and lattice distortion. In previous paper [12] we analyzed, in the (broken symmetry) Hartree-Fock approximation (HFA), one of the paradigmatic models of correlated electrons - the so-called $t-W$ model of spinless fermions with repulsive interaction $W$. In this contribution we study a simple paradigmatic model of electron-lattice interactions - the molecular crystal (MC) model [14, 15] in the static limit, and we contrast results for both models. The MC model describes electrons coupled to intramolecular (crystal field) vibrations [14, 15], and its Hamiltonian has the following form:

$$
\begin{aligned}
\hat{H} & =\sum_{i j \sigma} t_{i j} c_{i \sigma}^{+} c_{j \sigma}+\frac{1}{\sqrt{N}} \sum_{i q v} A_{q v}^{E} \mathrm{e}^{\mathrm{i} \boldsymbol{q} \cdot \boldsymbol{R}_{i}} n_{i} \hat{\varphi}_{q v} \\
& +\sum_{q v} \Omega_{q v} b_{q v}^{+} b_{q v}-(\mu-E) \sum_{i \sigma} n_{i \sigma}, \\
n_{\mathrm{e}} & =\frac{N_{\mathrm{e}}}{N}=\frac{1}{N} \sum_{i \sigma}\left\langle n_{i \sigma}\right\rangle, \quad 0<n_{\mathrm{e}}<2,
\end{aligned}
$$

where $c_{i \sigma}^{+}\left(c_{i \sigma}\right)$ are the creation (annihilation) operators for fermions on site $i, n_{i \sigma}=c_{i \sigma}^{+} c_{i \sigma}, t_{i j}$ are the single particle hopping integrals (between the nearest neighbors $t$ and the next nearest neighbors $\left.t_{2}\right), \mu$ - the chemical potential, $A_{q v}^{E}$ terms describe the coupling of elec-

\footnotetext{
* corresponding author; e-mail: czart@amu.edu.pl
}

trons to various types of intramolecular (or cation ligand) vibrations via modulation of the molecular (crystal field) energy $E, \Omega_{q v}$ are the phonon branches, arising from these intramolecular (cation ligand) vibrations [14], $b_{q v}^{(+)}$are phonon operators of the $v$-th phonon branch, $\hat{\varphi}_{q v}=b_{q v}+b_{q v}^{+}$. We are focusing on systems with adiabatic phonons $\left(\Omega_{q v} \ll t\right)$ and not too large electronphonon coupling where polaronic effects are negligible. We restrict considerations to the static limit and assume that the macroscopic distortions are caused by phonon modes with $q=Q(Q$ - half the smallest reciprocal lattice vector). Therefore we put [14]:

$$
\hat{\varphi}_{q v} \rightarrow \sqrt{N} \varphi_{q v} \delta_{|q|, Q}
$$

neglecting all phonons with $q \neq Q$ and determining the classical field $\varphi_{Q v}$ by minimizing of the free energy. We have performed extensive studies of the phase diagrams and thermodynamic properties of the charge orderings in the $t-W$ and the MC models for arbitrary particle concentration and various lattice structures, including: $d=2$ square lattice, $d=3$ sc lattice and the hypercubic lattice of infinite dimension $(d=\infty)$ [15]. In this contribution we concentrate on the problem of phase separations (PS) involving $\mathrm{CO}$ and the effects of next-nearest-neighbor hopping $\left(t_{2}\right)$ on the charge ordered states, which have not been studied until now for the static limit of the MC model, and compare the results with those obtained for the $t-W$ model [12]. The case of half filled band $\left(n_{\mathrm{e}}=1\right)$ as well as the cases of arbitrary particle concentrations $\left(0<n_{\mathrm{e}}<2\right)$ are considered. Below we shortly summarize the main findings presenting selected phase diagrams which have been evaluated for a few representative cases at $T=0$ and $T>0$, by comparing the free energies of the homogeneous phases $(\mathrm{CO}, \mathrm{NO})$ and the PS states. 


\section{Results and discussion}

In the case of $d=2$ sq lattice and $d=3$ sc lattice we use the following notation: $G=\frac{G_{Q}^{E}}{t}, b=t_{2} / t$, where $G_{Q}^{E}=\sum_{v} \frac{4\left(A_{Q v}^{E}\right)^{2}}{\Omega_{Q v}}$. In the case of $d=\infty$ hypercubic lattice we have to rescale the hopping parameters $[11,16]$ to ensure finite variance of free electron kinetic energy and the denotations are: $G=\frac{G_{Q}^{E}}{t^{*}}, b=t_{2}^{*} / t^{*}$, where $t^{*}$, $t_{2}^{*}$ are defined by $t=t^{*} / \sqrt{2 d}, t_{2}=t_{2}^{*} / \sqrt{2 d(d-1)}$. The definitions of $W_{0}$ for the $t-W$ model are given in [12].

The $\mathrm{CO}$ phases are characterized by the electron $\mathrm{CO}$ parameter:

$$
\Delta_{Q}^{\mathrm{e}}=\frac{1}{N} \sum_{i \sigma}\left\langle n_{i \sigma}\right\rangle \mathrm{e}^{\mathrm{i} \boldsymbol{Q} \cdot \boldsymbol{R}_{i}} \neq 0,
$$

and simultaneously by the static internal distortions

$$
\varphi_{Q v}=-\frac{2 A_{Q v}^{E} \Delta_{Q}^{\mathrm{e}}}{\Omega_{Q v}} \neq 0 .
$$

Obviously, the following relation also holds:

$$
\Delta_{Q}^{\mathrm{e}}=-\sum_{v} 2 A_{Q v}^{E} \varphi_{Q v} / G_{Q}^{E} .
$$

\subsection{The phase diagrams in the absence of frustration} $\left(t_{2}=0\right)$

At half-filling, i.e. for $n_{\mathrm{e}}=1$ for $\mathrm{MC}$ model and $n_{\mathrm{s}}=$ 0.5 in case of the $t-W$ model [12], the ground state (GS) of both models for $d \geq 2$ hypercubic lattices is charge ordered for any interaction strength $G, W_{0}>0$.

Beyond half-filling, in all these cases the GS diagrams for both models consist of two states: the nonordered metal (NO) if the interaction parameter ( $G$ for the $\mathrm{MC}$ model and $W_{0}$ for the $t-W$ model) is smaller than a critical value for a given concentration $n$ and the phase separated state: $\mathrm{CO} / \mathrm{NO}$, if the interaction parameter (IP) is greater than a critical value (see Figs. 1a,b in this paper and Figs. 1a,b in [12], plotted for sq and $d=\infty$ lattices). The transitions at the critical value of the IP for both models are discontinuous (of the 1st order) for any $n$, and the critical value strongly increases with the increase of the deviation from half-filling. A qualitative difference between the models exists in the case of sq lattice: for the $\mathrm{MC}$ model there exists a finite critical value of the IP for which the system remains in the $\mathrm{PS}(\mathrm{CO} / \mathrm{NO})$ state in the whole range of concentrations $n$ (except half-filling) (Fig. 1a), while for the $t-W$ model there is no such critical value of the IP (Fig. 1a in [12]). In the case of $d=\infty$ lattice there is just a quantitative difference between the models: the area of a phase diagram occupied by the $\mathrm{PS}(\mathrm{CO} / \mathrm{NO})$ state is much larger on the GS diagram of the MC model (Fig. 1b) than on the GS diagram of the $t-W$ model (Fig. 1b in [12]).

With increasing temperature the systems discussed can exhibit several different types of behavior. For the MC model one can observe either: (i) a sequence of two transitions $\mathrm{PS}(\mathrm{CO} / \mathrm{NO}) \rightarrow \mathrm{CO} \rightarrow \mathrm{NO}$, or (ii) a single 1st order transition PS $\rightarrow$ NO, or (iii) a single 2nd order

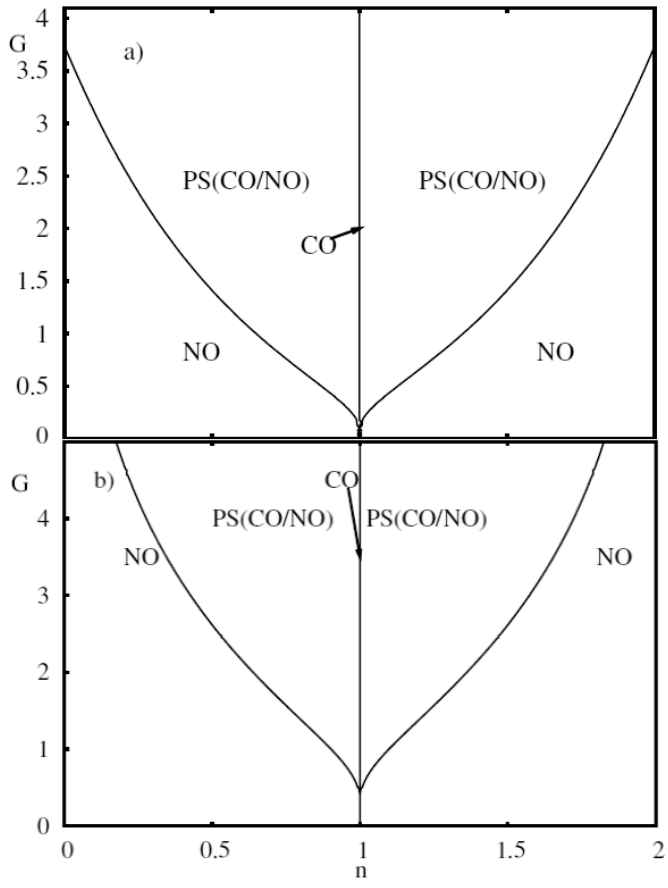

Fig. 1. Ground state phase diagrams of the MC model in the static limit as a function of $n$ for $t_{2}=0$. The phase separated region is marked $\mathrm{PS}(\mathrm{CO} / \mathrm{NO})$. The boundary between $\mathrm{PS}(\mathrm{CO} / \mathrm{NO})$ and nonordered state (NO) is of the first order (a) for $d=2$ sq lattice; (b) for $d=\infty$. The pure CO is stable at $n=1.0$ only.

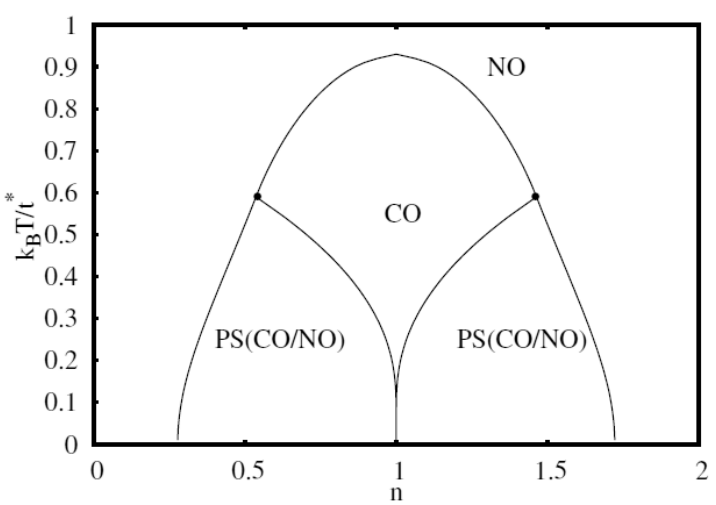

Fig. 2. Finite temperature phase diagram plotted for $d=\infty, t_{2}=0$ for interaction parameter $G^{*}=4.0$. Transitions between $\mathrm{PS}(\mathrm{CO} / \mathrm{NO})$ state and $\mathrm{NO}, \mathrm{CO}$ phases are of the first order; transition between $\mathrm{CO}$ and NO phases are of the second order. Filled dots denote the tricritical points (TCP).

transition $\mathrm{CO} \rightarrow \mathrm{NO}$ (at half-filling only). In Fig. 2 we show the $T-n$ phase diagram of the MC model for $d=\infty$ lattice and a representative value of $G$. The diagram is qualitatively different from the analogous diagram for the $t-W$ model (Fig. 2 in [12]). Whereas on the diagram for the $t-W$ model in definite ranges of $n$ and $W_{0}$ one finds the reentrant charge-order phenomena, on the diagram of 
the $\mathrm{MC}$ model the $\mathrm{PS}(\mathrm{NO} / \mathrm{CO})$ state extends over much larger area of a phase space, preventing the reentrant $\mathrm{CO}$ transition.

\subsection{The effects of frustration $\left(t_{2} \neq 0\right)$}

As in the case of the $t-W$ model, the frustration introduced by $t_{2}$ in the MC model at half-filling suppresses the perfect nesting instability towards $\mathrm{CO}$ phases at weak interactions strength and the resulting GS diagrams strongly depend on the lattice dimensionality (see Figs. 3a,b and Fig. 3a in [12], plotted for sq and $d=\infty$ lattices). For both models at half-filling and $t_{2} \neq 0$ one
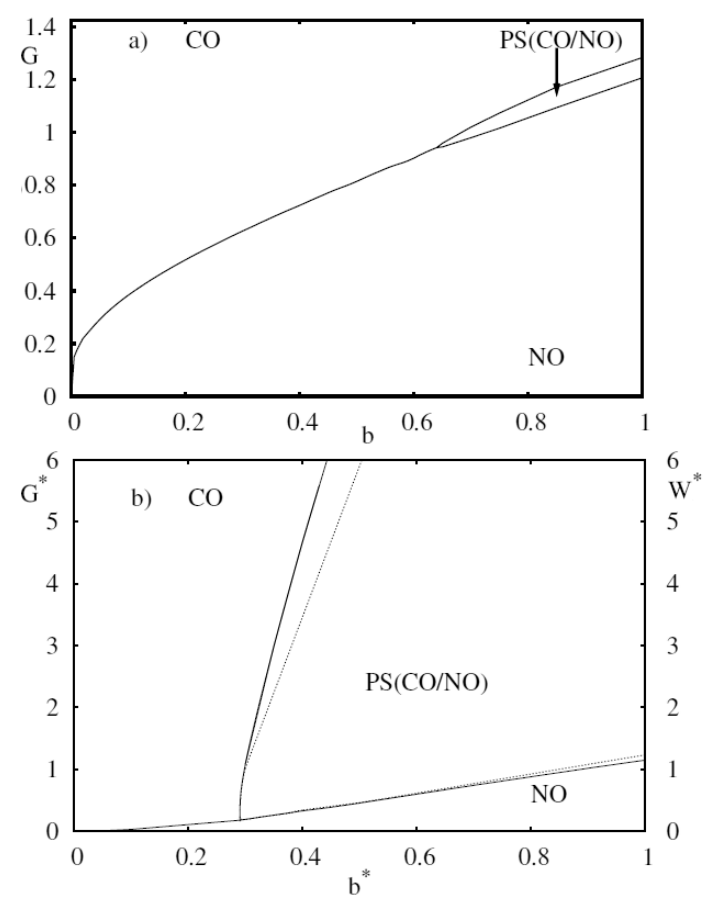

Fig. 3. Ground state phase diagrams at half-filling. (a) $d=2$ sq lattice, the MC model, (b) $d=\infty$ hypercubic lattice, the MC model - solid lines, the $t-W$ model - dashed lines. Transitions between $\mathrm{PS}(\mathrm{CO} / \mathrm{NO})$ state and $\mathrm{NO}, \mathrm{CO}$ phases are of the first order; transition between $\mathrm{CO}$ and $\mathrm{NO}$ phases are of the second order.

can observe several types of behavior, depending on IP, $b$ and lattice dimensionality, in particular: (i) a single second order transition: $\mathrm{NO} \rightarrow \mathrm{CO}$. It occurs e.g. for the $t-W$ model for sq lattice and $b<0.085$. (ii) a sequence of two 1st order transitions $\mathrm{NO} \rightarrow \mathrm{PS}(\mathrm{CO} / \mathrm{NO}) \rightarrow \mathrm{CO}$. It occurs e.g. for both models for $d=\infty$ lattice and large $b^{*}$. $A$ qualitative difference between the models exists in the case of sq lattice in the presence of large frustration: for the MC model there is a sequence of two 1st order transitions $\mathrm{NO} \rightarrow \mathrm{PS}(\mathrm{CO} / \mathrm{NO}) \rightarrow \mathrm{CO}$ (Fig. 3a), while for the $t-W$ model there is a single second order transition NO $\rightarrow$ CO for large $b$ (Fig. 3a in [12]). In the case of $d=\infty$ lattice (Fig. $3 \mathrm{~b}$ ) there is just a quantitative difference between the models: the area of a phase diagram occupied by the $\mathrm{PS}(\mathrm{CO} / \mathrm{NO})$ state is slightly larger on the GS diagram of the MC model than on the diagram of the $t-W$ model. We should point out that the previous results (Fig. 3b in [12]) reported for the $t-W$ model were calculated without considering possibility of the PS states and here we have supplemented those calculations.

As concerns the effects of frustration beyond half-filling, we have focused our studies mainly on the case of $d=2$ sq and $d=\infty$ lattices. More detailed discussion of this subject will be given elsewhere [15]. Let us only point that the structure of the diagrams is substantially changed in comparison to that obtained for $t_{2}=0$. In particular: (i) for $t_{2} \neq 0$ in definite ranges of $n$ and IP there can exist at $T=0$ an extended area of the homogeneous CO phase, (ii) frustration breaks electron-hole symmetry, and calculated phase diagrams are often qualitatively different for electron and hole doping.

\section{Conclusions}

We have presented results for the MC model in the static limit considering both the homogeneous phases $(\mathrm{NO}, \mathrm{CO})$ and the $\mathrm{PS}(\mathrm{CO} / \mathrm{NO})$ states, for $d=2 \mathrm{sq}$ and $d=\infty$, non-frustrated $\left(t_{2}=0\right)$ and frustrated $\left(t_{2} \neq 0\right)$ lattices, at representative fillings and temperatures. We have found various first and second order phase transitions, possible sequences of transitions and critical points. The phase diagrams strongly depend on the lattice dimensionality and frustration. We also compared results for the MC model with the analogous results for the $t-W$ model. Generally, we may conclude that the $\mathrm{PS}(\mathrm{CO} / \mathrm{NO})$ solutions are favored by the MC model Hamiltonian in comparison with the $t-W$ model Hamiltonian, as it is clearly seen on corresponding phase diagrams of both models. Finally, let us remark that we have restricted our study to the two-sublattice $\mathrm{CO}$ case and we have not considered a possible incommensurate ordering. We leave this problem for the future investigation.

\section{References}

[1] M. Imada, A. Fujimori, Y. Tokura, Rev. Mod. Phys. 70, 1039 (1998).

[2] T. Kimura, R. Kumai, Y. Tokura, J.Q. Li, Y. Matsui, Phys. Rev. B 58, 11081 (1998); E. Dagotto, T. Hotta, A. Moreo, Phys. Rep. 344, 1 (2001).

[3] T. Goto, B. Lüthi, Adv. Phys. 52, 67 (2003).

[4] M.Yu. Kagan, K.I. Kugel, Phys. Usp. 44, 553 (2001); M.Yu. Kagan, K.I. Kugel, D.I. Khomskii, J. Exp. Theor. Phys. 93, 415 (2001).

[5] R. Micnas, J. Ranninger, S. Robaszkiewicz, Rev. Mod. Phys. 62, 113 (1990).

[6] S. Robaszkiewicz, G. Pawłowski, Acta Phys. Pol. A 90, 569 (1996).

[7] P. Fulde, P. Thalmeir, G. Zwicknagel, Solid State Phys. 60, 1 (2006).

[8] Y.Z. Zhang, M.T. Tran, V. Yushankhai, P. Thalmeier, Eur. Phys. J. B 44, 265 (2005); A.T. Hoang, P. Thalmeier, J. Phys., Condens. Matter 14, 6639 (2002). 
[9] K. Rościszewski, A.M. Oleś, J. Phys., Condens. Matter 15, 8363 (2003).

[10] N.H. Tong, S.-Q. Shen, R. Bulla, Phys. Rev. B 70, 085118 (2004).

[11] G.S. Uhrig, R. Vlaming, Phys. Rev. Lett. 71, 271 (1993).

[12] W. Czart, S. Robaszkiewicz, B. Tobijaszewska, Acta Phys. Pol. A 114, 73 (2008).
[13] H. Seo, J. Merino, H. Yoshioka, M. Ogata, J. Phys. Soc. Jpn. 75, 51009 (2006).

[14] S. Robaszkiewicz, T. Kostyrko, Physica B 112, 389 (1982); 121, 134 (1983).

[15] W.R. Czart, P.R. Grzybowski, S. Robaszkiewicz, in preparation.

[16] E. Müller-Hartmann, Z. Phys. B 74, 507 (1989); W. Metzner, D. Vollhardt, Phys. Rev. Lett. 62, 324 (1989). 